Fecha de recepción: marzo 2020 Fecha de aceptación: abril 2020 Versión final: mayo 2020

\section{Prólogo. La Imagen personal frente a los nuevos desafíos 2020}

María Pía Estebecorena ${ }^{(1)}$

Resumen: Los cambios globales en la economía y la "nueva normalidad" como forma de vida diaria hacen que el concepto de Imagen esté cambiando hacia el encuentro de una nueva ubicación que permita dar respuesta frente a nuevas variables con las que se enfrenta cada persona.

La percepción del tiempo, la distancia sin distancias, la virtualidad de la imagen define una nueva forma de significar el concepto Imagen.

Sin duda esto afecta también no sólo lo perceptible sino la forma de estructurar la enseñanza de aspectos tales como el Color, cuando se creía que era imposible enseñarlo o diagnosticarlo virtualmente con la misma calidad con las que se aplica las técnicas en forma presencial.

Todas estas variables conforman un nuevo paradigma que marca un nuevo rumbo dentro de la línea de investigación conceptual en Imagen $360^{\circ}$ que comenzamos a desarrollar en 2019 Diversos autores y expertos en Imagen de países con culturas y costumbres tan disímiles como India, Austria, México, Brasil y Argentina nos comparten en esta nueva edición de Cuadremos Académicos de la UP los nuevos desafíos que tienen al 2020 como punto de partida para que podamos repensar conceptualmente la Imagen dentro de este contexto con final abierto.

Palabras clave: Imagen 360 - Nueva normalidad - COVID19 - Imagen Política - Psicología de la Imagen - Marca Personal - Millenialls - Habilidades Blandas - Concepto de Imagen integral - Marketing - Negocios - Sustentabilidad.

[Resúmenes en inglés y portugués en las páginas 15-16]

(1) María Pía Estebecorena es Lic. en Ciencia Política, Asesora de Imagen. Cuenta con Posgrados en Psicología Positiva, Estrategia, Geopolítica, Retail Marketing \& Loyalty (CRM). Certificada internacionalmente en Incubadora de Negocios por la Universidad de Salamanca y Doinglobal, Sillicon Valley. Es Point of You Trainer dentro del área de Coaching. Actualmente es parte del Directorio Internacional de AICI como VP Business Development y VP President de AICI Argentina Interior Central Chapter.

Fundó AICI Capitulo Argentina en 2011 y AICI Capitulo Argentina Interior Central en 2020. Fue Membership Chair of Latin America \& Spain for AICI Global, Past President de AICI Argentina por dos períodos. Trabajó como Directora de Negocios y Product Manager por más de 16 años para: Falabella, Walmart, Tiendas Paris de Chile y estuvo a 
cargo del start up de Elektra de Argentina del Grupo Salinas de México. En el año 2011 UNESCO la distinguió como Embajadora de Paz. Especialista en Personal Branding, Management and Professional Image. Speaker internacional, dando capacitaciones por todo Latinoamérica, USA e India. En el 2017 obtuvo la Certificación Internacional en Imagen por AICI Internacional, AICI CIC y en 2019 la Certificación AICI CIP. A cargo de CEImagen, Capacitación y Entrenamiento en Imagen y Personal Branding desde 2001

Los cambios globales en la economía y la "nueva normalidad" como forma de vida diaria hacen que el concepto de Imagen esté cambiando hacia el encuentro de una nueva ubicación que permita dar respuesta frente a nuevas variables con las que se enfrenta cada persona.

La percepción del tiempo, la distancia sin distancias, la virtualidad de la imagen define una nueva forma de significar el concepto Imagen.

Sin duda esto afecta también no sólo lo perceptible sino la forma de estructurar la enseñanza de aspectos tales como el Color, cuando se creía que era imposible enseñarlo o diagnosticarlo virtualmente con la misma calidad con las que se aplica las técnicas en forma presencial.

Todas estas variables conforman un nuevo paradigma que marca un nuevo rumbo dentro de la línea de investigación conceptual en Imagen $360^{\circ}$ que comenzamos a desarrollar en 2019. Diversos autores y expertos en Imagen de países con culturas y costumbres tan disímiles como India, Austria, México, Brasil y Argentina nos comparten en esta nueva edición de Cuadremos Académicos de la UP los nuevos desafíos que tienen al 2020 como punto de partida para que podamos repensar conceptualmente la Imagen dentro de este contexto con final abierto.

En todos los trabajos que hemos desarrollado aquí el eje conceptual tenemos como objetivo invitar al lector a poder definir qué es la Imagen como concepto hoy, luego que la pandemia ha atravesado la idea establecida de comunicación, de asistir a una clase, de compartir un espacio social en un evento. Todo eso hoy se ha transformado hoy.

El distanciamiento social, involucrar tecnologías para poder comunicarnos, nuestro propio lenguaje no verbal, el cual aún se ve comprometido por elementos que son parte de esta nueva realidad como el tapabocas. Todo este conjunto de nuevas normas, la facilidad con que asimilamos la adaptación y nos abrimos a nuevos estándares sociales, definen además un componente básico que es parte de nuestra imagen interna que es la resiliencia personal.

Es decir, en cuestión de meses, la idea que desarrollamos en el Cuaderno Académico 97 acerca del concepto de Imagen $360^{\text {a }}$ a partir de la cual tomábamos una visión integradora para definir de un modo más actual el concepto de Imagen, fue confirmada.

El COVID y todo su entorno de nuevas reglas y usos modificaron la forma de percibir la imagen interna y externa de los otros y redefinir nuestra propia imagen integral. Quién mostramos ser, cómo elegimos comunicarnos a través de una plataforma digital compartida, qué exteriorizamos de nosotros en cada imagen, en cada video. 
Hay un cambio de consciencia sobre la percepción de la propia imagen al que fuimos arrastrados todos, incluidas aquellas personas que orgullosamente decían no estar en las redes sociales, quienes se resistían a compartir un video, a grabar su voz. Todos debimos aprender la importancia de cuidar un fondo en una foto o durante una trasmisión porque lo que se muestra en la pantalla es la extensión de nuestra imagen hacia los lugares que habitamos con nuestra propia importa personal

Aun los que eligen un fondo digital están comunicando mucho de su propia imagen.

Aspectos antes considerados menores como el tono de voz, la entonación, la oratoria, pasa hoy a ser un elemento fundamental por ejemplo para un profesor frente a una clase online. La persuasión personal, la ortografía en un chat compartido en vivo, las reglas hacia los demás en una transmisión por Instagram o Facebook, el nivel de respeto por el disenso, formas de gestionar los conflictos.

Las habilidades blandas ${ }^{1}$ son otro emergente aspecto junto con la Marca Personal que en los últimos diez años ha cobrado protagonismo. A medida que aumenta la exposición de la imagen personal, va creciendo el espectro que abarca el concepto Imagen, movilizado por la demanda de comunicar mejor al multiplicarse los espacios de enlace y participación social. La Imagen como tal hoy está atada necesariamente a una estrategia de marketing personal, política, social, corporativa y/o de marca. Constituye una alianza indisoluble que le posibilita un direccionamiento de acuerdo a la elaboración de un propósito.

Pensar todos estos aspectos como componentes de la imagen era algo sin duda osado y desafiante hasta el punto de generar incredulidad en el año 2000 cuando apenas comenzaba a trabajarse la idea de Imagen dentro de las capacitaciones y en la enseñanza.

Todo esto nos demuestra y confirma que lo que creíamos entender como imagen en el 2000 hoy representa sólo el entre un 30 y un $40 \%$ de la imagen total según estudios de últimas mediciones ${ }^{2}$.

Con esto me refiero a aspectos externos de la Imagen como la vestimenta, estilos, proporciones ópticas y uso del guardarropa.

En el presente trabajo, destacados profesionales dentro de AICI nos muestran de qué forma aparecen estos cambios a la hora de abordar las asesorías en diferentes y exceden las fronteras culturales, sociales y geográficas para desembocar en un común denominador que encierra este nuevo concepto integral de Imagen $360^{\mathrm{a}}$

La experta en Imagen Política, Coca Sevilla, AICI CIM de México abre esta línea de investigación compartiendo su visión acerca de los cambios que afectaron este nicho a partir del COVID19 y de qué forma impacta en nuestra imagen social todas las nuevas medidas $\mathrm{y}$ accesorios que van formando parte de nuestra nueva realidad. El entorno parece volverse complejo repentinamente y nuevos desafíos más radicales aparecen para los expertos en Imagen Política.

Sonia Dubey Dewan, AICI CIP de India, en paralelo con Sevilla, desarrolla las variaciones en el concepto de Marca Personal que corre los objetivos en el marketing personal y explica las nuevas fases por las cuales pasa hoy la buena gestión de la marca y la importancia destacada que adquiere en la vida profesional. Nos acerca desde un país muy remoto su experiencia y nos enriquece con aspectos globales aplicables a culturas occidentales.

Eva Koeck Eripek,AICICIM de Austria expone todos estos cambios conceptuales plasmados en la práctica del asesoramiento de imagen para políticos ygrupos especiales. En su trabajo se 
desarrollaun pormenorizadoanálisis delosaspectospsicológicosquesearticulan con un propósito específicocomofacilitadoresdela comunicación públicaylaalta exposición en medios. Políticos, artistas y personas influyentes son quienes demandan más rápidamente estas técnicas en la construcción de su comunicación.

Estos conceptos que van variando tan velozmente requieren de un momento de ser vueltos a analizar, pensar y reformular en lo que entendemos hoy por Imagen. En mi desarrollo del tema destaco las variables que intervienen este cambio y cómo se va gestionando a través de nuevos contenidos en la enseñanza dentro del aula y en la responsabilidad profesional individual de cada profesional en Imagen de actualizar sus competencias frente a un mercado con otras nuevas demandas

Desde India, esta misma idea es abordada por Nehaa Malhotra, AICI CIP. En su trabajo, describe la forma en que podemos integrar en la educación profesional estos conceptos con un valor agregado altamente actual: de qué forma agregar sustentabilidad a la asesoría de imagen. A priori se presenta como el nuevo desafío dentro de la profesión, en línea con el desarrollo de empresas B dentro de un contexto conceptual de economía circular.

Siguiendo con este recorrido, Fernanda Lucchesi AICI CIC, desde Brasil realiza un análisis de las competencias que hoy requiere nuestra profesión dentro de esta nueva economía hacia la cual el mundo se encamina más fuertemente en este momento y explica, como experta asesora de profesionales en Imagen de todo el mundo, cuál es la razón por la cual es habitual que no estén desarrolladas las capacidades emprendedoras sustentables del negocio y propone un cambio a partir del análisis de diversos relevamientos en el mercado. Para poder ir a la raíz de todos estos cambios económicos debemos comprender el cambio de mentalidad y de macro visión que traen las nuevas generaciones de Millenials. Rachel Jordán, AICI CIC desde Brasil hace visible los desafíos que representa este segmento en la sociedad actual. Una mirada extendida hacia las habilidades blandas justamente por ser el aspecto en donde más dificultades encuentran dentro del dialogo entre generaciones.

Ana Cheong Cheok Yin, AICI CIP y Aida Ainun Bahardin desde Malasia, en un estudio desarrollado para la Universidad Tunku Abdul Rahman desarrollan el aspecto psicológico de la Imagen dentro de este concepto integral en la mirada 360a, analizando los aspectos ocultos y los más reconocibles dentro de esta visión. En su trabajo, destacan punto por punto cada uno de los elementos que compone y cuál es el desarrollo de las nuevas técnicas en el análisis de imagen que se van aplicando al cliente.

Dentro de este cambio en cuanto a técnicas que lleva implícito el nuevo concepto de Imagen, cerramos la presente investigación con Luciana Ullrich de Brasil, experta en colorimetría y técnicas de color que suma su aporte desde lo técnico a partir de los contrastes trabajados desde la armonía y el impacto en el color aplicado a la imagen.

Deseo agradecer y destacar el esfuerzo que muchos de las profesionales que aquí participan han hecho frente a la situación de pandemia internacional. Desde muchos de los países que aquí participan la situación fue compleja en el primer semestre de este año. Pese a todo y a ser un año excepcional, el compromiso profesional de ellas fue inalterable. Vaya mi especial reconocimiento y agradecimiento en pos de la colaboración académica y docente de cada una. Finalmente, un reconocimiento a AICI Internacional, organización que certifica y agrupa en todo el mundo a profesionales en Imagen, al cual tengo el placer 
de pertenecer. AICI marca altos estándares en nuestra profesión los cuales son compartidos con gran compromiso académico para quienes participamos de la presente edición

\title{
Notas
}

1. Bustamante, Lilian. La importancia de las habilidades blandas y la Imagen Cuaderno Académico nro. 97 Universidad de Palermo.

2. Universidad Austral, Relaciones Interpersonales a partir de los datos de la encuesta CERI-UA, Argentina, mayo 2020.

\begin{abstract}
The global changes in the economy and the "new normality" as a daily way of life mean that the concept of Image is changing towards finding a new location that allows us to respond to new variables that each person is facing.

The perception of time, distance without distances, the virtuality of the image defines a new way of meaning the image concept.

Without a doubt this also affects not only the perceivable but the way of structuring the teaching of aspects such as Color, when it was believed that it was impossible to teach or diagnose it with virtually the same quality with which the techniques are applied in person.

All these variables make up a new paradigm that marks a new direction within the line of conceptual research in $360^{\circ}$ Image that we began to develop in 2019 Diverse authors and experts in Image from countries with cultures and customs as dissimilar as India, Austria, Mexico, Brazil and Argentina share in this new edition of UP Academic Cuadernos the new challenges that have 2020 as a starting point so that we can rethink the Image conceptually in this context with an open end.
\end{abstract}

Keywords: $360^{\circ}$ image - New normality - COVID19 - Political Image - Image Psychology - Personal brand - Millenialls - Soft skills - Integral Image Concept - Marketing - Business - Sustainability.

Resumo: As mudanças globais na economia e a "nova normalidade" como um modo de vida diário significam que o conceito de Imagem está mudando para encontrar um novo local que nos permita responder às novas variáveis que cada pessoa enfrenta.

A percepção do tempo, distância sem distância, a virtualidade da imagem define uma nova maneira de significar o conceito de imagem.

Sem dúvida, isso também afeta não apenas a percepção, mas a maneira de estruturar o ensino de aspectos como a Cor, quando se acreditava que era impossível ensiná-lo ou diagnosticá-lo com virtualmente a mesma qualidade com que as técnicas são aplicadas pessoalmente. 
Todas essas variáveis compõem um novo paradigma que marca uma nova direção dentro da linha de pesquisa conceitual em 360 Imagem que começamos a desenvolver em 2019 Diversos autores e especialistas em imagem de países de culturas e costumes tão diferentes quanto Índia, Áustria, México, Brasil e a Argentina compartilham nesta nova edição da UP Academic Cuadernos os novos desafios que têm 2020 como ponto de partida, para que possamos repensar a Imagem conceitualmente neste contexto com um final aberto.

Palavras chave: Imagem $360^{\circ}$ - Nova normalidade - COVID-19 - Imagem Política - Psicologia da Imagem - Marca pessoal - Millenialls - Habilidades interpessoais - Conceito de imagem integral - Marketing - O negócio - Sustentabilidade.

[Las traducciones de los abstracts fueron supervisadas por el autor de cada artículo] 